\title{
Optimal Constant-Stress Accelerated Degradation Test Plans Using Nonlinear Generalized Wiener Process
}

\author{
Zhen Chen, Shuo Li, and Ershun Pan \\ Department of Industrial Engineering \& Management, Shanghai Jiao Tong University, 800 Dongchuan RD, \\ Minhang District, Shanghai 200240, China
}

Correspondence should be addressed to Ershun Pan; pes@sjtu.edu.cn

Received 11 April 2016; Accepted 26 July 2016

Academic Editor: David Bigaud

Copyright (c) 2016 Zhen Chen et al. This is an open access article distributed under the Creative Commons Attribution License, which permits unrestricted use, distribution, and reproduction in any medium, provided the original work is properly cited.

Accelerated degradation test (ADT) has been widely used to assess highly reliable products' lifetime. To conduct an ADT, an appropriate degradation model and test plan should be determined in advance. Although many historical studies have proposed quite a few models, there is still room for improvement. Hence we propose a Nonlinear Generalized Wiener Process (NGWP) model with consideration of the effects of stress level, product-to-product variability, and measurement errors for a higher estimation accuracy and a wider range of use. Then under the constraints of sample size, test duration, and test cost, the plans of constantstress ADT (CSADT) with multiple stress levels based on the NGWP are designed by minimizing the asymptotic variance of the reliability estimation of the products under normal operation conditions. An optimization algorithm is developed to determine the optimal stress levels, the number of units allocated to each level, inspection frequency, and measurement times simultaneously. In addition, a comparison based on degradation data of LEDs is made to show better goodness-of-fit of the NGWP than that of other models. Finally, optimal two-level and three-level CSADT plans under various constraints and a detailed sensitivity analysis are demonstrated through examples in this paper.

\section{Introduction}

Reliability assessment is a critical task for improving the products' quality and reliability. Traditional life tests only record time-to-failure data to assess the lifetime distribution of the products. In presence of the quick development of technology and more and more demands from customers, some products need to be designed for highly reliable performance. Therefore, no failures are likely to occur over a reasonable test time and we would not obtain enough time-to-failure data. In such a case, if there exists a quality characteristic whose degradation is associated with the reliability of a product, we could obtain more information by collecting degradation data [1]. However, some highly reliable products degrade very slowly and it is impossible to access their lifetime within a reasonable test time. To overcome this problem, the accelerated degradation test (ADT) is usually adopted to collect the quality characteristic data under higher stress levels. The failure is defined as the event that the degradation path exceeds a fixed threshold.

In a common constant-stress ADT (CSADT), a number of units are allocated to several stress levels, and the degradation process is measured, analyzed, and extrapolated to the failure threshold in order to estimate the lifetime of the products under normal operating conditions. Consequently, before conducting an ADT, the degradation model and test plan should be developed first. The Wiener Process is often used for modelling ADT data because of its physical interpretations, nonmonotonic property, and infinite divisibility property, which could fit the products' dynamic characteristic well. Reference [2] gave an example of modelling the degradation process by the Wiener Process. Reference [3] studied the Wiener Process for handling the degradation data of LEDs.

Setting stress levels is an important part of designing an ADT. Several types of stress levels ADT have been proposed including CSADT, step-stress ADT (SSADT), and 
progressive-stress ADT (PSADT). Reference [4] designed a CSADT with a reciprocal Weibull degradation rate. Reference [5] proposed optimal SSADT plans for Gamma degradation processes, while [6] proposed optimal SSADT plans for Wiener degradation processes. Reference [7] discussed PSADT for highly reliable products under nonlinear Wiener Process. As the failure mechanisms of some products may drift along with stress increasing in SSADTs and PSADTs, we cannot use a stable model to describe the degradation paths. Thus CSADTs tend to be used more in practical applications. Moreover, if the CSADT had set only one stress level as in [4], no enough effective degradation data can be collected and the lifetime distribution of products cannot be estimated precisely [8].

In this article, a Nonlinear Generalized Wiener Process (NGWP) model is proposed to describe the degradation paths of LEDs. Next, a CSADT with the constraints of prefixed budget, test duration, and sample size is developed by minimizing the asymptotic variance of the estimated mean time to failure (MTTF) of the products under normal operation conditions. Then an optimization algorithm is used to determine the stress levels, the number of units allocated to each level, inspection frequency, and measurement times simultaneously. Finally, a comparison is conducted for measuring the goodness-of-fit of various Wiener Process models, and optimal two-level and three-level CSADT plans under various constraints are obtained.

The rest of this paper proceeds as follows. A motivating example and literature review for this study are provided in Section 2. Section 3 develops a NGWP model, derives the lifetime distribution, and proposes a maximum likelihood estimation (MLE) method to estimate the unknown parameters of the model. Section 4 presents the optimization model for CSADT plan. An example is presented to illustrate the proposed method in Section 5. Finally, Section 6 concludes the paper.

\section{Motivating Example and Literature Review}

2.1. Motivating Example. Nowadays, light-emitting diodes (LEDs) have been applied to many fields (e.g., traffic signals and full color displays) and are desirable, because of their high brightness, low power consumption, and high reliability. A LED fails when the LED relative luminosity drops to 0.5 , that is, $50 \%$ of initial luminosity. Hamada et al. [9] give a degradation data of relative luminosity (proportion of initial luminosity for LEDs). The data consist of three accelerating levels of thermal stress, $25^{\circ} \mathrm{C}, 65^{\circ} \mathrm{C}$, and $105^{\circ} \mathrm{C}$. At each level, the light intensity of 25 LEDs was measured at 29 inspection times. Figure 1 shows the degradation of light intensity of the LEDs.

Obviously, the degradation paths are nonlinear and the light intensity degrades more slowly at a lower thermal stress level. Reference [10] used the LED degradation data to optimize sample allocation for ADT based on Wiener Process. The Wiener Process, which modelled the degradation paths in [10], only took nonlinearity and the effects of stress level into account. However, Figure 1 indicates that the degradation paths of different products are also different. It means that the product-to-product variability among different products exists, owing to variation of materials, manufacturing, and environment [11]. Models with random effects can be used to represent the product-to-product variability $[12,13]$. Moreover, it is inevitable that some measurement errors may be introduced during the imperfect observation process in practical applications, and the external environment will also result in measurement errors [14]. The degradation models in $[12,13]$ did not take measurement errors into consideration, while the degradation model in [14] did not consider productto-product variability and the effects of stress level. Therefore, for modelling the degradation paths of LEDs more accurately and generally, a generalized Wiener Process model with a consideration of all these influence factors is urgently required for their potential importance.

In this paper, the NGWP is developed. The first advantage is that the NGWP which considers nonlinearity, the effects of stress level, product-to-product variability, and measurement errors simultaneously has higher estimation accuracy and better goodness-of-fit. The second advantage is that the NGWP covers the constant models as its special case. In practical applications, the specific form of the model can be selected according to the actual situation. Clearly, the NGWP can describe more complex and diverse degradation processes of many products and can be widely applied to highly reliable products.

Furthermore, if the NGWP is used to model the degradation paths of a CSADT, then how to conduct the test should be investigated. For this problem, there exist some issues worthy of further consideration:

(i) Typically, the asymptotic variance of the estimated MTTF (Avar) of the lifetime distribution of the product is used to judge whether a CSADT plan is optimal. So how can we derive the Avar of the NGWP?

(ii) Can we possibly determine the stress levels, the number of units allocated to each level, inspection frequency, and measurement times simultaneously, by optimizing the CSADT plan with the constraints on sample size, test duration, and test cost?

2.2. Literature Review. For the ADT modelling, [15] used the nonlinear Wiener Process to model PSADT, and [8] used the nonlinear Wiener Process to optimize sample size allocation for ADT. In addition, [12, 16, 17] discussed the residual life estimation based on nonlinear Wiener Process. Reference [13] investigated nonlinear Wiener Process with random effects for ADT data. So the nonlinear Wiener Process has an excellent applicability. Reference [14] showed that the nonlinear Wiener Process with measurement errors can be widely used to describe the degradation processes of various products. Similarly, [18] also indicated that the nonlinear Wiener Process with measurement errors performed better than other models in degradation data analysis. Furthermore, $[11,15,19]$ predicted the real time remaining useful life based on the nonlinear Wiener Process with measurement errors. 


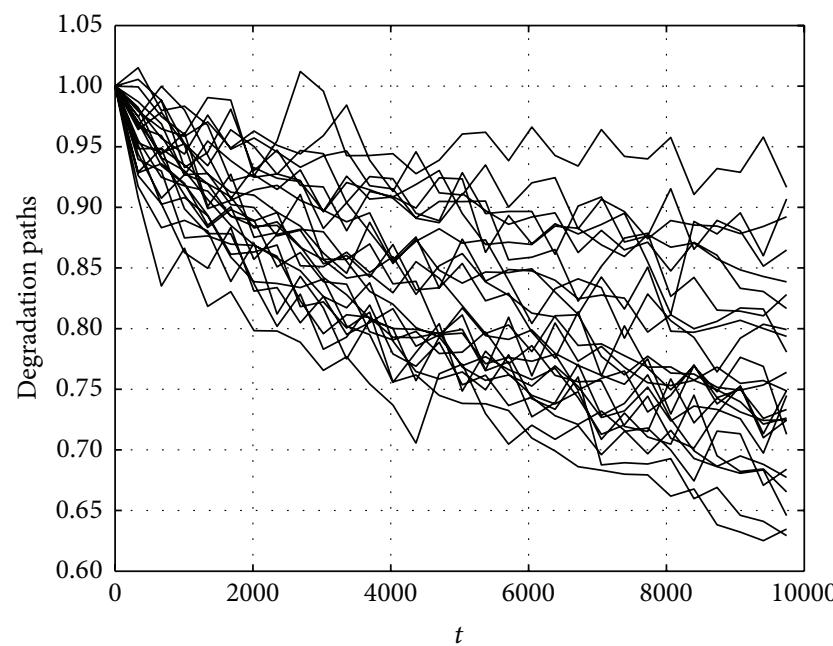

(a)

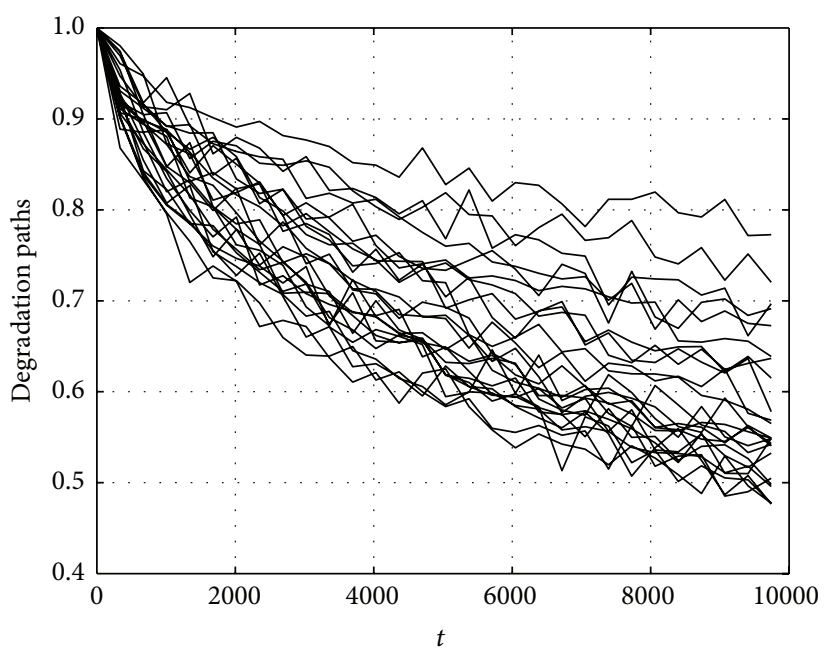

(b)

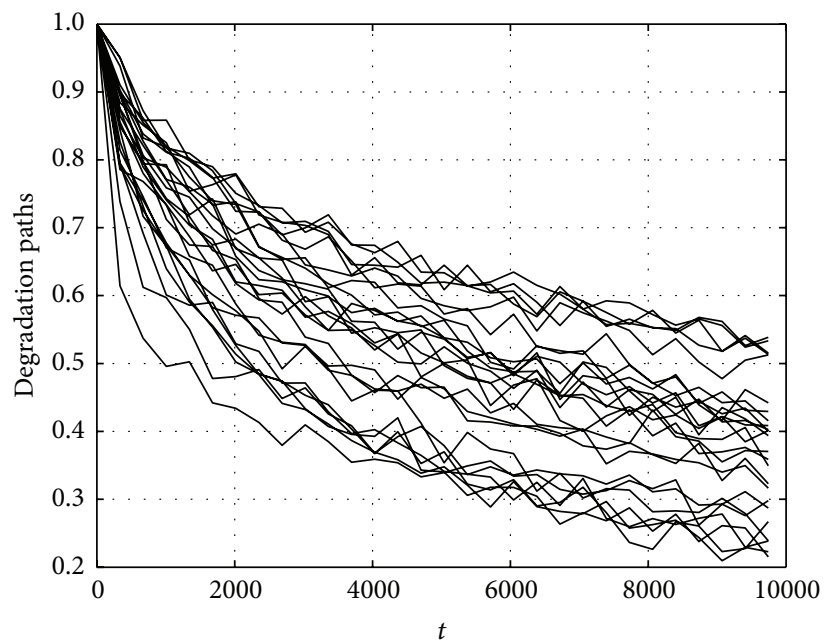

(c)

Figure 1: Plot of LED luminosity data over time $t$ in hours at (a) $25^{\circ} \mathrm{C}$, (b) $65^{\circ} \mathrm{C}$, and (c) $105^{\circ} \mathrm{C}$.

Reference [20] presented an accelerated-stress acceptance test based nonlinear Wiener Process.

Reference [21] developed an algorithm for determination of inspection frequency and unit allocation of ADT plans with multiple stress levels. Reference [22] set several stress levels to fix the stress levels and the number of samples for each stress level of ADT plans. Reference [23] developed an ADT method of luminous flux degradation for LEDs. Reference [24] suggested an analytical optimal CSADT design method for reliability demonstration by minimizing the asymptotic variance of decision variable in reliability demonstration under the constraints of sample size, test duration, test cost, and predetermined decision risks. To conduct a CSADT or SSADT, [25] determined the optimal decision variables based on C/D/A-optimality criteria. Reference [26] planed constant-stress accelerated life tests for acceleration model selection. Reference [27] established an optimal ADT procedure to minimize the asymptotic variance of the MLE of the MTTF of a product, given a budget for the total cost.

\section{Degradation Process Description}

3.1. Acceleration Degradation Process Modelling. To solve the problem about degradation model raised above, we propose the NGWP model considering the effects of stress level, product-to-product variability, and measurement errors, as follows:

$$
Y\left(t \mid S_{k}\right)=\eta_{k} \Lambda(t)+\sigma_{B} B(\Lambda(t))+\sigma_{\varepsilon} \varepsilon,
$$

where $\eta_{k}$ is the drift parameter related to the stress level $S_{k}, \sigma_{B}$ is the diffusion parameter, $\Lambda(t)$ is the drift function, $B(t)$ is the standard Brownian motion representing a timecorrelated structure, $\varepsilon$ is the measurement error with $N(0,1)$, and $\sigma_{\varepsilon}$ is the error coefficient.

Referring to (1), the drift parameter $\eta_{k}$ reflects the effect of stress level $S_{k}$ on the performance, and it determines the 
degradation rate of the NGWP. Acceleration model [28] in CSADT for products is commonly assumed as

$$
\eta_{k}=\alpha \exp \left(-\beta \varphi\left(S_{k}\right)\right)
$$

where $\alpha$ and $\beta$ are unknown coefficients and $\varphi\left(S_{k}\right)$ is a function of $S_{k}$. If $\varphi\left(S_{k}\right)=\ln S_{k}$, acceleration model is inverse power law. If $\varphi\left(S_{k}\right)=1 / S_{k}$, it is the Arrhenius equation. Besides, to account for the random variation of the performance caused by variation of materials, manufacturing, and environment, the drift parameter $\eta_{k}$ is assumed as a random parameter and it is s-independent from stress levels. Then, we have

$$
\eta_{k}=\alpha \exp \left(-\beta \varphi\left(S_{k}\right)\right), \quad \alpha \sim N\left(\mu_{\alpha}, \sigma_{\alpha}^{2}\right) .
$$

Note that if $\Lambda(t)=t$ in (1), then the NGWP model turns to the Linear Wiener Process. If $\sigma_{\alpha}^{2}$ in (3) is set to 0 , then the random model turns to the conventional acceleration model. This is as expected since any properly developed model should cover the constant model as its special case. Obviously, the NGWP model can describe more complex and diverse degradation processes of many products and have a wider range of applicability.

3.2. Derivation of the Lifetime Distribution. Let $X\left(t \mid S_{k}\right)=$ $\eta_{k} \Lambda(t)+\sigma_{B} B(\Lambda(t))$ denote the true degradation path of the product under stress $\left(S_{k}\right)$; then the product's lifetime $T$ can be defined as the first passage time when $X\left(t \mid S_{k}\right)$ crosses the critical value $\omega$ under a normal operation stress $\left(S_{0}\right)$. Hence, we have

$$
T=\inf \left\{t \mid X\left(t \mid S_{0}\right) \geq \omega\right\} .
$$

The lifetime $T$ conditioning on $\eta_{0}$ follows a transformation-inverse Gaussian distribution, whose cumulative distribution function (CDF) is

$$
\begin{aligned}
F_{T}(t)= & \Phi\left(\frac{\eta_{0} \Lambda(t)-\omega}{\sqrt{\sigma_{B}^{2} \Lambda(t)}}\right) \\
& +\exp \left\{\frac{2 \omega \eta_{0}}{\sigma_{B}^{2}}\right\} \Phi\left(-\frac{\eta_{0} \Lambda(t)+\omega}{\sqrt{\sigma_{B}^{2} \Lambda(t)}}\right), \quad t>0,
\end{aligned}
$$

where $\Phi(\cdot)$ is the CDF of the standard normal distribution.

Considering the accelerated model in (3), that is, $\eta_{k}=$ $\alpha \exp \left(-\beta \varphi\left(S_{k}\right)\right), \alpha \sim N\left(\mu_{\alpha}, \sigma_{\alpha}^{2}\right)$, the CDF of $T$ by integrating $\eta_{0}$ out of (5) becomes

$$
\begin{aligned}
& F_{T}(t)=\Phi\left(\frac{\mu_{\alpha} e^{-\beta \varphi\left(S_{k}\right)} \Lambda(t)-\omega}{\sqrt{\sigma_{B}^{2} \Lambda(t)+\sigma_{\alpha}^{2} e^{-2 \beta \varphi\left(S_{k}\right)} \Lambda(t)^{2}}}\right) \\
&+ \exp \left(\frac{2 \mu_{\alpha} e^{-\beta \varphi\left(S_{k}\right)} \omega}{\sigma_{B}^{2}}+\frac{2 \sigma_{\alpha}^{2} e^{-2 \beta \varphi\left(S_{k}\right)} \omega^{2}}{\sigma_{B}^{4}}\right) \\
& \cdot \Phi\left(-\frac{\left(2 \sigma_{\alpha}^{2} e^{-2 \beta \varphi\left(S_{k}\right)} \omega \Lambda(t)+\sigma_{B}^{2}\left(\mu_{\alpha} e^{-\beta \varphi\left(S_{k}\right)} \Lambda(t)+\omega\right)\right)}{\sigma_{B}^{2} \sqrt{\sigma_{B}^{2} \Lambda(t)+\sigma_{\alpha}^{2} e^{-2 \beta \varphi\left(S_{k}\right)} \Lambda(t)^{2}}}\right) .
\end{aligned}
$$

From (6), the product's MTTF under the normal operation stress $\left(S_{0}\right)$ can be approximated by using

$$
T_{\mathrm{MTTF}}=E(T)=E\left(E\left(T \mid \eta_{0}\right)\right)=\frac{\omega}{\mu_{\alpha} e^{-\beta / S_{0}}} .
$$

3.3. Parameters Estimation. In this subsection, the issue of estimating the model parameter $\boldsymbol{\theta}=\left(\mu_{\alpha}, \sigma_{\alpha}, \beta, \sigma_{B}, \sigma_{\varepsilon}\right)$ is addressed by using the MLE method. Suppose that $N$ test units are available for conducting a CSADT under the following conditions:

(i) The CSADT uses $d$-stress levels, $\left(S_{0}\right) \leq S_{1} \leq \cdots \leq S_{d}$.

(ii) We assign $n_{k}$ items for a degradation test at a stress level $S_{k}$, where $\sum_{k=1}^{d} n_{k}=N, 1 \leq k \leq d$.

(iii) For each stress level, the inspections are made $m$ times and the measurements of each unit are available at time $t_{1}, t_{2}, \ldots, t_{m}$

(iv) The inspection frequency is $f$ and satisfies $f=t_{j}-$ $t_{j-1}, 1 \leq j \leq m, t_{0}=0$. Thus $t_{m}=m f$.

(v) For $1 \leq i \leq n_{k}, 1 \leq k \leq d, 1 \leq j \leq m$, let $Y_{i}\left(t_{j} \mid S_{k}\right)$ denote the sample degradation path of $i$ th test unit at time $t_{j}$ under the stress level $S_{k}$.

$$
Y_{i j k}=Y_{i}\left(t_{j} \mid S_{k}\right)=\eta_{k} \Lambda\left(t_{j}\right)+\sigma_{B} B\left(\Lambda\left(t_{j}\right)\right)+\sigma_{\varepsilon} \varepsilon_{i j k},
$$

where the measurement errors $\varepsilon_{i j k}$ are assumed to be i.i.d. realizations of $\varepsilon$.

(vi) The Arrhenius equation is adopted to describe the relationship between $S_{k}$ and $\eta_{k}$ :

$$
\eta_{k}=\alpha \exp \left(\frac{-\beta}{S_{k}}\right), \quad \alpha \sim N\left(\mu_{\alpha}, \sigma_{\alpha}^{2}\right) .
$$

For simplicity, let $\Lambda=\left(\Lambda\left(t_{1}\right), \ldots, \Lambda\left(t_{m}\right)\right)^{\prime}, \mathbf{Y}_{i k}=$ $\left(Y_{i}\left(t_{1} \mid S_{k}\right), \ldots, Y_{i}\left(t_{m} \mid S_{k}\right)\right)^{\prime}$, and $\mathbf{Y}=\left(\mathbf{Y}_{11}^{\prime}, \ldots, \mathbf{Y}_{n_{1} 1}^{\prime}, \mathbf{Y}_{12}^{\prime}, \ldots\right.$, $\left.\mathbf{Y}_{n_{2} 2}^{\prime}, \ldots, \mathbf{Y}_{1 d}^{\prime}, \ldots, \mathbf{Y}_{n_{d} d}^{\prime}\right)^{\prime}$. Clearly, $\mathbf{Y}_{i k}$ follows a multivariate normal distribution with mean $\mu_{\alpha} e^{-\beta / S_{k}} \boldsymbol{\Lambda}$ and variance $\boldsymbol{\Sigma}_{k}=$ $\sigma_{\alpha}^{2} e^{-2 \beta / S_{k}} \boldsymbol{\Lambda} \boldsymbol{\Lambda}^{\prime}+\boldsymbol{\Omega}$, where $\boldsymbol{\Omega}=\sigma_{B}^{2} \mathbf{Q}+\sigma_{\varepsilon}^{2} \mathbf{I}_{m}, \mathbf{I}_{m}$ is an identity matrix of order $m$, and

$$
\mathbf{Q}=\left[\begin{array}{cccc}
\Lambda\left(t_{1}\right) & \Lambda\left(t_{1}\right) & \cdots & \Lambda\left(t_{1}\right) \\
\Lambda\left(t_{1}\right) & \Lambda\left(t_{2}\right) & \cdots & \Lambda\left(t_{2}\right) \\
\vdots & \vdots & \ddots & \vdots \\
\Lambda\left(t_{1}\right) & \Lambda\left(t_{2}\right) & \cdots & \Lambda\left(t_{m}\right)
\end{array}\right]
$$

The log-likelihood function of $\boldsymbol{\theta}$ is

$$
\begin{aligned}
& \ell(\boldsymbol{\theta} \mid \mathbf{Y})=-\frac{N m \ln (2 \pi)}{2}-\sum_{k=1}^{d} \frac{n_{k}}{2} \ln \left|\mathbf{\Sigma}_{k}\right|-\frac{1}{2} \\
& \cdot \sum_{k=1}^{d} \sum_{i=1}^{n_{k}}\left(\mathbf{Y}_{i k}-\mu_{\alpha} e^{-\beta / S_{k}} \boldsymbol{\Lambda}\right)^{\prime} \Sigma_{k}^{-1}\left(\mathbf{Y}_{i k}-\mu_{\alpha} e^{-\beta / S_{k}} \boldsymbol{\Lambda}\right),
\end{aligned}
$$


where $\left|\boldsymbol{\Sigma}_{k}\right|=|\boldsymbol{\Omega}|\left(1+\sigma_{\alpha}^{2} e^{-2 \beta / S_{k}} \boldsymbol{\Lambda}^{\prime} \boldsymbol{\Omega}^{-1} \boldsymbol{\Lambda}\right)$, and $\boldsymbol{\Sigma}_{k}{ }^{-1}=\boldsymbol{\Omega}^{-1}-$ $\left(\sigma_{\alpha}^{2} e^{-2 \beta / S_{k}} /\left(1+\sigma_{\alpha}^{2} e^{-2 \beta / S_{k}} \boldsymbol{\Lambda}^{\prime} \boldsymbol{\Omega}^{-1} \boldsymbol{\Lambda}\right)\right) \boldsymbol{\Omega}^{-1} \boldsymbol{\Lambda} \boldsymbol{\Lambda}^{\prime} \boldsymbol{\Omega}^{-1}$.

By differentiating the log-likelihood function in (11) with respect to $\mu_{\alpha}$, we have

$$
\begin{aligned}
\frac{\partial \ell(\boldsymbol{\theta} \mid \mathbf{Y})}{\partial \mu_{\alpha}}= & \sum_{k=1}^{d} \sum_{i=1}^{n_{k}} e^{-\beta / S_{k}} \boldsymbol{\Lambda}^{\prime} \boldsymbol{\Sigma}_{k}^{-1} \mathbf{Y}_{i k} \\
& -\mu_{\alpha} \sum_{k=1}^{d} \sum_{i=1}^{n_{k}} e^{-2 \beta / S_{k}} \Lambda^{\prime} \boldsymbol{\Sigma}_{k}^{-1} \boldsymbol{\Lambda}
\end{aligned}
$$

For specified values of $\sigma_{\alpha}, \beta, \sigma_{B}$, and $\sigma_{\varepsilon}$, the MLE of $\mu_{\alpha}$ can be obtained by equaling (12) to 0 ; that is,

$$
\widehat{\mu}_{\alpha}=\frac{\sum_{k=1}^{d} \sum_{i=1}^{n_{k}} e^{-\beta / S_{k}} \Lambda^{\prime} \Sigma_{k}^{-1} \mathbf{Y}_{i k}}{\sum_{k=1}^{d} \sum_{i=1}^{n_{k}} e^{-2 \beta / S_{k}} \Lambda^{\prime} \Sigma_{k}^{-1} \Lambda} .
$$

Then, the profile log-likelihood function of $\sigma_{\alpha}, \beta, \sigma_{B}$, and $\sigma_{\varepsilon}$ can be given by substituting $\widehat{\mu}_{\alpha}$ for $\mu_{\alpha}$ in (12). Subsequently, the MLEs of $\sigma_{\alpha}, \beta, \sigma_{B}$, and $\sigma_{\varepsilon}$ can be obtained by maximizing the profile log-likelihood function through a multipledimensional search. Here, we made use of the MATLAB function "fmincon" for this purpose. By substituting the MLE of $\sigma_{\alpha}, \beta, \sigma_{B}$, and $\sigma_{\varepsilon}$ into (13), the MLE of $\mu_{\alpha}$ can be obtained.

Finally, we obtain all MLEs of the model's unknown parameters, $\widehat{\boldsymbol{\theta}}=\left(\widehat{\mu}_{\alpha}, \widehat{\sigma}_{\alpha}, \widehat{\beta}, \widehat{\sigma}_{B}, \widehat{\sigma}_{\varepsilon}\right)$. The MLE of MTTF under a normal operation stress $\left(S_{0}\right)$ is

$$
\widehat{T}_{\text {MTTF }}=\frac{\omega}{\widehat{\mu}_{\alpha} e^{-\widehat{\beta} / S_{0}}} .
$$

\section{The Optimal CSADT Plans}

In this section, to design an efficient CSADT for a typical highly reliable product, we consider the optimization problem of determining the allocation of the units $\left(n_{1}, \ldots, n_{d}\right)$, inspection frequency $(f)$, and measurement times $(m)$ by minimizing the asymptotic variance of $\widehat{T}_{\text {MTTF }}$ under normal operating conditions subject to a prefixed budget.

4.1. Objective Function. From (14), we find that the value of $\widehat{T}_{\text {MTTF }}$ determines the accuracy of extrapolation. The smaller asymptotic variance of $\widehat{T}_{\text {MTTF }}$ is, the more efficient CSADT plan will be. Thus, we set the asymptotic variance of $\widehat{T}_{\text {MTTF }}$ as an objective function by using the delta method

$$
\operatorname{Avar}\left(\widehat{T}_{\text {MTTF }} \mid \boldsymbol{\xi}\right)=\mathbf{H}^{\prime} \mathbf{I}^{-1}(\boldsymbol{\theta}) \mathbf{H},
$$

where $\boldsymbol{\xi}=\left(n_{1}, \ldots, n_{d}, f, m\right), \mathbf{H}^{\prime}=\left(\partial T_{\mathrm{MTTF}} / \partial \mu_{\alpha}, 0, \partial T_{\mathrm{MTTF}} /\right.$ $\partial \beta, 0,0)$, and $\mathbf{I}(\boldsymbol{\theta})$ is the Fisher information matrix. The detailed expressions for calculating $\operatorname{Avar}\left(\widehat{T}_{\mathrm{MTTF}}\right)$ are listed in the Appendix.

4.2. Constraints. The constraints in the design of CSADT plan usually include the following:

(i) The test time $t_{m}$ should not exceed the specified test duration $t_{A}$. (ii) The sample size should not exceed the number of test units available $N_{A}$.

(iii) The total test cost TC should not exceed the prefixed budget $C_{b}$.

The total cost of conducting a CSADT can be expressed as

$$
\begin{aligned}
\mathrm{TC}\left(n_{1}, \ldots, n_{d}, f, m\right)= & C_{\text {op }} f m+C_{\text {mea }} \sum_{k=1}^{d} n_{k} m \\
& +C_{d} \sum_{k=1}^{d} n_{k},
\end{aligned}
$$

where $C_{\text {op }}$ denotes the unit cost for operation per time, $C_{\text {mea }}$ denotes the unit cost for each measurement, $C_{d}$ denotes the unit cost for each test device.

4.3. Optimization Model. From the expressions given above, the optimization problem can be formulated as follows:

$$
\begin{aligned}
& \text { Min } \quad \operatorname{Avar}\left(\widehat{T}_{\mathrm{MTTF}} \mid \xi\right) \\
& \text { Subject to } \operatorname{TC}\left(n_{1}, \ldots, n_{d}, f, m\right) \leq C_{b}, \\
& t_{m} \leq t_{A}, N \leq N_{A},
\end{aligned}
$$

where $\boldsymbol{\xi}=\left(n_{1}, \ldots, n_{d}, f, m\right) \in N^{d+2}$.

Due to the complex form of the objective function, an analytic expression for solution of this problem seems impossible. However, with the simplicity in the structure of the constraint and the integer restriction on these decision variables, the optimal solution $\xi^{*}=\left(n_{1}^{*}, \ldots, n_{d}^{*}, f^{*}, m^{*}\right)$ can be easily determined by a complete enumeration method in a finite number of steps. The detailed algorithm is described below in nine steps.

Step 1. Set $m_{\max }=\min \left(\left\lfloor\left(C_{b}-C_{d e} \times d\right) /\left(C_{\text {mea }} \times d+\right.\right.\right.$ $\left.\left.\left.C_{\text {op }}\right)\right\rfloor,\left\lfloor t_{A} / f\right\rfloor\right)$, where $\lfloor\cdot\rfloor$ is a truncated integer; $m_{\max }$ is the largest possible number for $m$, when $f=1$, and $n_{k}=1$ for $1 \leq k \leq d$.

Step 2. Set $m=2$.

Step 3. Set $f_{\max }=\min \left(\left\lfloor\left(C_{b}-d \times C_{\text {mea }} \times m-d \times C_{d e}\right) /\left(C_{\text {op }} \times\right.\right.\right.$ $\left.m)\rfloor,\left\lfloor t_{A} / m\right\rfloor\right) ; f_{\max }$ is the largest possible number for $f$ when $n_{k}=1$ for $1 \leq k \leq d$ and fixed $m$.

Step 4. Set $f=1$.

Step 5. Find $n_{1}, \ldots, n_{d} \in N$ such that $\mathrm{TC}\left(n_{1}, \ldots, n_{d}, f, m\right) \leq$ $C_{b}, \sum_{k=1}^{d} n_{k} \leq N_{A}$.

Step 6. Calculate Avar $\left(\widehat{T}_{\text {MTTF }} \mid \boldsymbol{\xi}\right)$ by $\boldsymbol{\xi}$.

Step 7. Set $f=f+1$, and repeat Steps 5 and 6 until $f=f_{\max }$.

Step 8. Set $m=m+1$, and repeat Steps 3 and 7 until $m=$ $m_{\max }$. 
TABLE 1: Estimated parameters of the degradation model.

\begin{tabular}{lccccc}
\hline$\widehat{\mu}_{\alpha}$ & $\widehat{\sigma}_{\alpha}$ & $\widehat{\beta}$ & $\widehat{\sigma}_{B}$ & $\widehat{\sigma}_{\varepsilon}$ & $\ell / 10^{3}$ \\
\hline 1.2889 & 0.3120 & 1831.7 & 0.0071 & 0.0270 & 4.2416 \\
\hline
\end{tabular}

Step 9. The optimal solution $\xi^{*}=\left(n_{1}^{*}, \ldots, n_{d}^{*}, f^{*}, m^{*}\right)$ is then obtained as $\min _{\xi} \operatorname{Avar}\left(\widehat{T}_{\mathrm{MTTF}} \mid \xi\right)$.

\section{Illustrative Example}

In this section, we illustrate the proposed procedure with a numerical example based on the degradation data of LEDs. In order to capture the curvature by the NGWP model, the data of LEDs is a logarithm transformation and the general function $\Lambda(t)=t^{0.5}$. The transformation degradation paths which have clearly linear characteristic are presented in Figure 2. Then the parameters of the degradation model, that is, $\mu_{\alpha}, \sigma_{\alpha}, \beta, \sigma_{B}, \sigma_{\varepsilon}$, are estimated by the proposed method from the 75 LEDs, respectively, and shown in Table 1. By using the estimates, the optimal CSADT plans can be obtained.

5.1. Comparison with Other Degradation Models. For further illustrating the rationality and applicability of the proposed model, this subsection compares some degradation models with the degradation data of LEDs. For simplicity, the NGWP model in (1) is referred to as $M_{0}$.

$$
M_{0}: Y\left(t \mid S_{k}\right)=\eta_{k} \Lambda(t)+\sigma_{B} B(\Lambda(t))+\sigma_{\varepsilon} \varepsilon,
$$

where $\eta_{k}=\alpha \exp \left(-\beta \varphi\left(S_{k}\right)\right), \alpha \sim N\left(\mu_{\alpha}, \sigma_{\alpha}^{2}\right)$.

If the degradation path is assumed to be linear in (18), that is, $\Lambda(t)=t$, then the NGWP model turns into a Linear Wiener Process model.

$$
M_{1}: Y\left(t \mid S_{k}\right)=\eta_{k} t+\sigma_{B} B(t)+\sigma_{\varepsilon} \varepsilon,
$$

where $\eta_{k}=\alpha \exp \left(-\beta \varphi\left(S_{k}\right)\right), \alpha \sim N\left(\mu_{\alpha}, \sigma_{\alpha}^{2}\right)$.

Similarly, if the measurement errors are not considered, then the NGWP model becomes a Wiener Process model without measurement errors.

$$
M_{2}: Y\left(t \mid S_{k}\right)=\eta_{k} \Lambda(t)+\sigma_{B} B(\Lambda(t)),
$$

where $\eta_{k}=\alpha \exp \left(-\beta \varphi\left(S_{k}\right)\right), \alpha \sim N\left(\mu_{\alpha}, \sigma_{\alpha}^{2}\right)$.

If the degradation path is treated as a fixed-effect model, then the following Wiener Process model without random effects can be used to describe the LED degradation paths.

$$
M_{3}: Y\left(t \mid S_{k}\right)=\eta_{k} \Lambda(t)+\sigma_{B} B(\Lambda(t))+\sigma_{\varepsilon} \varepsilon,
$$

where $\eta_{k}=\alpha \exp \left(-\beta \varphi\left(S_{k}\right)\right)$ and $\alpha$ and $\beta$ are both constants.

To measure the goodness-of-fit of these different Wiener Process models above, the Akaike information criterion (AIC) is employed. AIC, which is frequently used in engineering and statistical literature for the purpose of model selection, is defined as

$$
\mathrm{AIC}=2 m-2 \ell,
$$

where $m$ is the number of model parameters and $\ell$ is the maximized value of the log-likelihood function of the estimated model. When there are several potential available models, the one with the smallest AIC among these could be selected as the best fitting model.

Table 2 shows the estimation results of the parameters, the log-likelihood function value, and the AIC. From Table 2, it can be found that the model $M_{0}$ obtains the highest $\ell$ and the lowest AIC compared to other models. This implies that the proposed model has better model fit than other models. Therefore, a model considering nonlinearity, the effects of stress level, product-to-product variability, and measurement errors simultaneously has a better and wider range of practical applicability.

5.2. Optimal CSADT Plans. We first consider a two-level CSADT plan $(d=2)$, where $S_{0}=20^{\circ} \mathrm{C}, S_{1}=40^{\circ} \mathrm{C}$, and $S_{2}=60^{\circ} \mathrm{C}$. And the lifetime $T$ is the first passage time when degradation path crosses $\omega=-\ln (0.5)$. Suppose $\left(C_{\text {op }}, C_{\text {mea }}, C_{d}\right)=(0.5,2,30)$; the optimal CSADT plans under various constraints which were determined by using the algorithm presented earlier are shown in Table 2. Since there are three constraints, the optimal plans are obtained by fixing two of the three and varying the last one. For example, Table 3(a) displays the optimal solutions by fixing the test duration and the sample size and varying the budget. When $\left(C_{b}, t_{A}, N_{A}\right)=(2500,2000,50)$, the optimal test plan turned out to be $\left(n_{1}^{*}, n_{2}^{*}, f^{*}, m^{*}\right)=(31,19,300,4)$. That is, the optimal sample sizes for stress level $S_{1}$ and $S_{2}$ are 31 and 19, respectively, and the total test time for the CSADT is 1200 hours. Under such a test plan, the total cost is 2500 .

It is interesting to observe that the three constraints all have a significant impact on the results. As one of them is increasing and the other two remain constant, the change of the optimal test plan is getting smaller and smaller. This result is true because a constraint will be out of action when it becomes lager enough. In this case, the optimal plan is completely determined by the other two constraints. Therefore, the constraints should be developed reasonably in practical application.

Moreover, the asymptotic variance of $\widehat{T}_{\text {MTTF }}$ was gradually decreasing. This means the test accuracy becomes higher with the constraints relaxing. Besides, we could observe that the magnitude of the reduction of $\operatorname{Avar}\left(\widehat{T}_{\mathrm{MTTF}} \mid \xi\right)$ was also getting smaller slowly. Therefore, we only need to select an appropriate constraints condition rather than a more relaxed one within the requirement of test accuracy.

5.3. Sensitivity Analysis. In practice, the estimated parameter $\widehat{\boldsymbol{\theta}}=\left(\widehat{\mu}_{\alpha}, \widehat{\sigma}_{\alpha}, \widehat{\beta}, \widehat{\sigma}_{B}, \widehat{\sigma}_{\varepsilon}\right)$ would depart from the true parameter $\boldsymbol{\theta}=\left(\mu_{\alpha}, \sigma_{\alpha}, \beta, \sigma_{B}, \sigma_{\varepsilon}\right)$. Without loss of generality, we assume that $\rho_{1}, \rho_{2}, \rho_{3}$ denote the estimation bias for $\mu_{\alpha}, \sigma_{\alpha}, \beta$ (as the values of $\widehat{\sigma}_{B}$ and $\widehat{\sigma}_{\varepsilon}$ are too small, we do not consider their estimation bias).

Under the same cost configuration $\left(C_{\text {op }}, C_{\text {mea }}, C_{d}, C_{b}\right.$, $\left.t_{A}, N_{A}\right)=(0.5,2,30,2500,2000,50)$, Table 4 displays the optimal plan under various combinations of $\widehat{\mu}_{\alpha}\left(1+\rho_{1}\right)$, $\widehat{\sigma}_{\alpha}\left(1+\rho_{2}\right)$, and $\widehat{\beta}\left(1+\rho_{3}\right)$. From these results, we can see that 
TABLE 2: Comparisons of four degradation models with the degradation data of LEDs.

\begin{tabular}{lcccccrr}
\hline & $\widehat{\mu}_{\alpha}$ & $\widehat{\sigma}_{\alpha}$ & $\widehat{\beta}$ & $\widehat{\sigma}_{B}$ & $\widehat{\sigma}_{\varepsilon}$ & $\ell$ & AIC \\
\hline$M_{0}$ & 1.2889 & 0.3120 & 1831.7 & 0.0071 & 0.0270 & $\mathbf{4 2 4 1 . 6}$ & $-\mathbf{8 4 7 3 . 2}$ \\
$M_{1}$ & 0.0150 & 0.0018 & 1878.9 & 0.0021 & 0.0160 & 3685.3 & -7360.6 \\
$M_{2}$ & 1.3781 & 0.0001 & 1853.1 & 0.026 & - & 3691.2 & -7374.4 \\
$M_{3}$ & 1.3629 & - & 1851.2 & 0.010 & 0.0260 & 4156.9 & -8305.8 \\
\hline
\end{tabular}

Remark 1: "-" means that the estimate does not exist in that case.

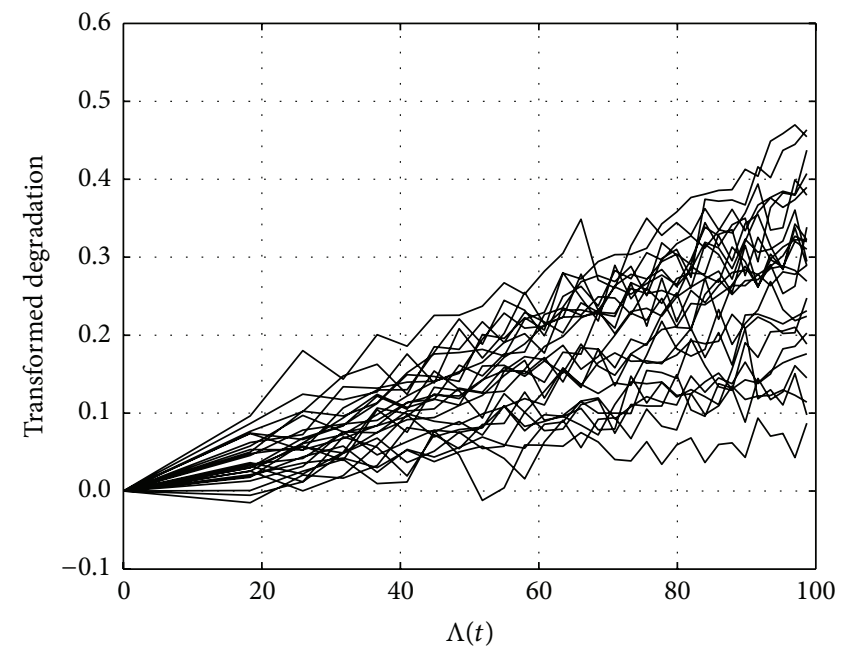

(a)

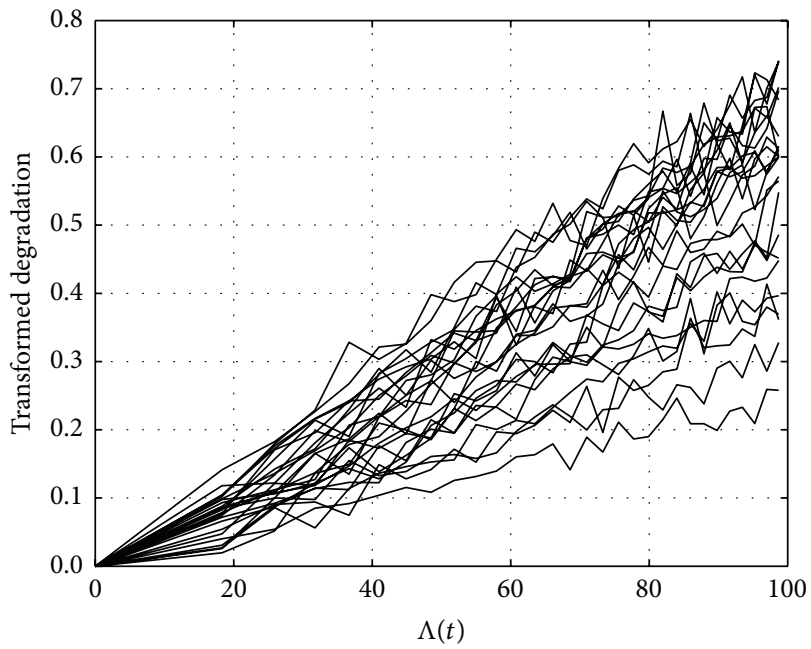

(b)

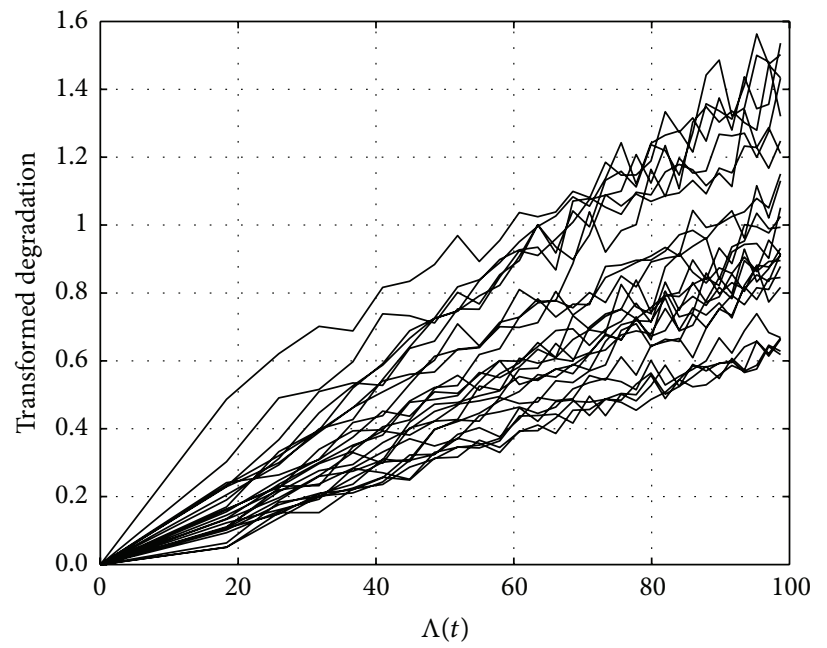

(c)

Figure 2: Plot of transformation degradation paths of LEDs luminosity data over time $\Lambda(t)=t^{0.5}$ in hours at (a) $25^{\circ} \mathrm{C}$, (b) $65^{\circ} \mathrm{C}$, and (c) $105^{\circ} \mathrm{C}$.

the optimal test plan $\left(n_{1}^{*}, n_{2}^{*}, f^{*}, m^{*}\right)$ tends to be robust to estimation bias, given that the bias is not too large.

5.4. Optimal CSADT Plans with $d$-Stress, Where $d \geq 3$. We have already presented the optimal CSADT plans with two stress levels. When stress levels $d \geq 3$, it is not easy to get results of test plans. Instead, we select a condition of three stress levels to show the optimal CSADT plans. Suppose three stress levels are $S_{1}=40^{\circ} \mathrm{C}, S_{2}=50^{\circ} \mathrm{C}$, and $S_{3}=60^{\circ} \mathrm{C}$. The cost configuration is $\left(C_{\text {op }}, C_{\text {mea }}, C_{d}\right)=(0.5,2,30)$. Table 5 lists the optimal CSADT plans under various constraints.

No matter how the constraints vary, the optimal plans demonstrate that the lowest stress levels were allocated to more units. Comparing Tables 3 and 5, we can find that the $\operatorname{Avar}\left(\widehat{T}_{\text {MTTF }} \mid \xi\right)$ of the optimal two-level CSADT plan is smaller than that of the optimal three-level CSADT plan under the same conditions. It means that the two-level CSADT plans have higher test accuracy. However, this result 
TABLE 3: (a) Optimal two-level CSADT plans under various prefixed budget constraints. (b) Optimal two-level CSADT plans under various duration constraints. (c) Optimal two-level CSADT plans under various sample size constraints.

(a)

\begin{tabular}{lcccccc}
\hline$C_{b}$ & $n_{1}^{*}$ & $n_{2}^{*}$ & $f^{*}$ & $m^{*}$ & Avar $/ 10^{3}$ & Budgets \\
\hline 1000 & 13 & 7 & 320 & 2 & 0.8933 & 1000 \\
1500 & 18 & 10 & 328 & 3 & 0.4805 & 1500 \\
2000 & 26 & 15 & 349 & 3 & 0.3212 & 1999.5 \\
2500 & 31 & 19 & 300 & 4 & 0.2363 & 2500 \\
3000 & 30 & 19 & 314 & 6 & 0.1987 & 3000 \\
3500 & 31 & 19 & 333 & 6 & 0.1921 & 3099 \\
4000 & 31 & 19 & 333 & 6 & 0.1921 & 3099 \\
\hline
\end{tabular}

Remark 2: the test duration constraint $t_{A}=2000$ and the sample size constraint $N_{A}=50$.

(b)

\begin{tabular}{lcccccc}
\hline$t_{A}$ & $n_{1}^{*}$ & $n_{2}^{*}$ & $f^{*}$ & $m^{*}$ & Avar $/ 10^{3}$ & Budgets \\
\hline 500 & 32 & 18 & 100 & 5 & 0.3001 & 2250 \\
1000 & 31 & 19 & 200 & 5 & 0.2382 & 2500 \\
1500 & 31 & 19 & 300 & 4 & 0.2363 & 2500 \\
2000 & 31 & 19 & 300 & 4 & 0.2363 & 2500 \\
2500 & 31 & 19 & 300 & 4 & 0.2363 & 2500 \\
3000 & 31 & 19 & 300 & 4 & 0.2363 & 2500 \\
\hline
\end{tabular}

Remark 3: the prefixed budget constraint $C_{b}=2500$ and the sample size constraint $N_{A}=50$.

(c)

\begin{tabular}{lcccccc}
\hline$N_{A}$ & $n_{1}^{*}$ & $n_{2}^{*}$ & $f^{*}$ & $m^{*}$ & Avar $/ 10^{3}$ & Budgets \\
\hline 20 & 12 & 8 & 85 & 23 & 0.4054 & 2497.5 \\
30 & 18 & 12 & 200 & 10 & 0.2974 & 2500 \\
40 & 25 & 15 & 211 & 7 & 0.2515 & 2498.5 \\
50 & 31 & 19 & 300 & 4 & 0.2362 & 2500 \\
60 & 32 & 19 & 281 & 4 & 0.2362 & 2500 \\
70 & 32 & 19 & 281 & 4 & 0.2362 & 2500 \\
\hline
\end{tabular}

Remark 4: the prefixed budget constraint $C_{b}=2500$ and the test duration constraint $t_{A}=2000$. does not intend to suggest that the optimal plans are the only choice for conducting a CSADT. A degradation test may need degradation data of more than two stress levels, so as to verify the validity of the model in (1) and ensure that the research can be generalized.

\section{Conclusion}

We have investigated the optimal CSADT plans based on the NGWP model. The NGWP model which considers nonlinearity, the effects of stress level, the product-to-product variability, and measurement errors has higher estimation accuracy and better goodness-of-fit. By minimizing the asymptotic variance of the reliability estimation of the products under normal operation conditions subject to sample size, test duration, and test cost, the objective of CSADT plans is to properly determine the stress levels, the number of units allocated to each level, inspection frequency, and measurement times, simultaneously. An optimization algorithm is proposed to determine the decision variables. Moreover, the MLE method to estimate unknown parameters and MTTF of products is presented in this study. Then, comparison based on degradation data of LEDs is conducted to show better goodness-of-fit of the NGWP than that of other models. Finally, optimal two-level CSADT plans and optimal three-level CSADT plans under various constraints are demonstrated. A detailed sensitivity analysis for the estimated parameters is also conducted in this study.

When the stress levels are more than three, a new algorithm which is more efficient should be developed. If the sample size is only moderate or even small, it is necessary to investigate other methods for designing a CSADT. Overall, many interesting issues about degradation models and accelerated test plans require further study.

\section{Appendix}

\section{Detailed Expressions of $\operatorname{Avar}\left(\widehat{T}_{\mathrm{MTTF}} \mid \boldsymbol{\xi}\right)$ and $\mathrm{I}(\boldsymbol{\theta})$ in (15)}

The expression of Fisher information $\mathbf{I}(\boldsymbol{\theta})$ is

$$
\mathbf{I}(\boldsymbol{\theta})=\left[\begin{array}{ccccc}
E\left(-\frac{\partial^{2} \ell}{\partial \mu_{\alpha}^{2}}\right) & E\left(-\frac{\partial^{2} \ell}{\partial \mu_{\alpha} \partial \sigma_{\alpha}}\right) & E\left(-\frac{\partial^{2} \ell}{\partial \mu_{\alpha} \partial \beta}\right) & E\left(-\frac{\partial^{2} \ell}{\partial \mu_{\alpha} \partial \sigma_{B}}\right) & E\left(-\frac{\partial^{2} \ell}{\partial \mu_{\alpha} \partial \sigma_{\varepsilon}}\right) \\
E\left(-\frac{\partial^{2} \ell}{\partial \sigma_{\alpha} \partial \mu_{\alpha}}\right) & E\left(-\frac{\partial^{2} \ell}{\partial \sigma_{\alpha}^{2}}\right) & E\left(-\frac{\partial^{2} \ell}{\partial \sigma_{\alpha} \partial \beta}\right) & E\left(-\frac{\partial^{2} \ell}{\partial \sigma_{\alpha} \partial \sigma_{B}}\right) & E\left(-\frac{\partial^{2} \ell}{\partial \sigma_{\alpha} \partial \sigma_{\varepsilon}}\right) \\
E\left(-\frac{\partial^{2} \ell}{\partial \beta \partial \mu_{\alpha}}\right) & E\left(-\frac{\partial^{2} \ell}{\partial \beta \partial \sigma_{\alpha}}\right) & E\left(-\frac{\partial^{2} \ell}{\partial \beta^{2}}\right) & E\left(-\frac{\partial^{2} \ell}{\partial \beta \partial \sigma_{B}}\right) & E\left(-\frac{\partial^{2} \ell}{\partial \beta \partial \sigma_{\varepsilon}}\right) \\
E\left(-\frac{\partial^{2} \ell}{\partial \sigma_{B} \partial \mu_{\alpha}}\right) & E\left(-\frac{\partial^{2} \ell}{\partial \sigma_{B} \partial \sigma_{\alpha}}\right) & E\left(-\frac{\partial^{2} \ell}{\partial \sigma_{B} \partial \beta}\right) & E\left(-\frac{\partial^{2} \ell}{\partial \sigma_{B}^{2}}\right) & E\left(-\frac{\partial^{2} \ell}{\partial \sigma_{B} \partial \sigma_{\varepsilon}}\right) \\
E\left(-\frac{\partial^{2} \ell}{\partial \sigma_{\varepsilon} \partial \mu_{\alpha}}\right) & E\left(-\frac{\partial^{2} \ell}{\partial \sigma_{\varepsilon} \partial \sigma_{\alpha}}\right) & E\left(-\frac{\partial^{2} \ell}{\partial \sigma_{\varepsilon} \partial \beta}\right) & E\left(-\frac{\partial^{2} \ell}{\partial \sigma_{\varepsilon} \partial \sigma_{B}}\right) & E\left(-\frac{\partial^{2} \ell}{\partial \sigma_{\varepsilon}^{2}}\right)
\end{array}\right],
$$


TABLE 4: Optimal CSADT plan considering the estimation bias for $\mu_{\alpha}, \sigma_{\alpha}, \beta$.

\begin{tabular}{lccccccccc}
\hline$\rho_{1}$ & $\rho_{2}$ & $\rho_{3}$ & $N_{A}$ & $n_{1}^{*}$ & $n_{2}^{*}$ & $f^{*}$ & $m^{*}$ & Avar/10 & Budgets \\
\hline $5 \%$ & $5 \%$ & $5 \%$ & 29 & 16 & 13 & 280 & 5 & 0.4925 \\
$5 \%$ & 0 & 0 & 31 & 19 & 12 & 300 & 4 & 0.1944 \\
$5 \%$ & $-5 \%$ & $-5 \%$ & 31 & 19 & 12 & 300 & 4 & 0.8060 \\
0 & $5 \%$ & 0 & 31 & 19 & 12 & 300 & 4 & 0.2485 & 2500 \\
0 & 0 & $-5 \%$ & 30 & 20 & 10 & 300 & 4 & 0.1045 \\
0 & $-5 \%$ & $5 \%$ & 29 & 16 & 13 & 280 & 5 & 0.5514 & 2500 \\
$-5 \%$ & $5 \%$ & $-5 \%$ & 30 & 20 & 10 & 300 & 4 & 0.1368 \\
$-5 \%$ & 0 & $5 \%$ & 29 & 17 & 12 & 264 & 5 & 0.7048 \\
$-5 \%$ & $-5 \%$ & 0 & 31 & 19 & 12 & 300 & 4 & 0.2759 \\
0 & 0 & 0 & 31 & 19 & 12 & 300 & 4 & 2500 \\
\hline
\end{tabular}

where

$$
\begin{aligned}
& E\left(-\frac{\partial^{2} \ell}{\partial \mu_{\alpha}^{2}}\right)=\sum_{k=1}^{d} n_{k} e^{-2 \beta / S_{k}} \Lambda^{\prime} \Sigma_{k}^{-1} \boldsymbol{\Lambda}, \\
& E\left(-\frac{\partial^{2} \ell}{\partial \mu_{\alpha} \partial \sigma_{\alpha}}\right)=E\left(-\frac{\partial^{2} \ell}{\partial \sigma_{\alpha} \partial \mu_{\alpha}}\right)=0, \\
& E\left(-\frac{\partial^{2} \ell}{\partial \mu_{\alpha} \partial \beta}\right)=E\left(-\frac{\partial^{2} \ell}{\partial \beta \partial \mu_{\alpha}}\right)=-\mu_{\alpha} \sum_{k=1}^{d} \sum_{i=1}^{n_{k}} \frac{e^{-2 \beta / S_{k}}}{S_{k}} \Lambda^{\prime} \Sigma_{k}^{-1} \boldsymbol{\Lambda}, \\
& E\left(-\frac{\partial^{2} \ell}{\partial \mu_{\alpha} \partial \sigma_{B}}\right)=E\left(-\frac{\partial^{2} \ell}{\partial \sigma_{B} \partial \mu_{\alpha}}\right)=0, \\
& E\left(-\frac{\partial^{2} \ell}{\partial \mu_{\alpha} \partial \sigma_{\varepsilon}}\right)=E\left(-\frac{\partial^{2} \ell}{\partial \sigma_{\varepsilon} \partial \mu_{\alpha}}\right)=0, \\
& E\left(-\frac{\partial^{2} \ell}{\partial \sigma_{\alpha}^{2}}\right)=\sum_{k=1}^{d} \frac{n_{k}}{2} \frac{\partial^{2}\left(\ln \left|\Sigma_{k}\right|\right)}{\partial \sigma_{\alpha}^{2}}+E\left[\frac{1}{2}\right. \\
& \left.\cdot \sum_{k=1}^{d} \sum_{i=1}^{n_{k}}\left(\mathbf{Y}_{i k}-\mu_{\alpha} e^{-\beta / S_{k}} \boldsymbol{\Lambda}\right)^{\prime} \frac{\partial^{2}\left(\boldsymbol{\Sigma}_{k}^{-1}\right)}{\partial \sigma_{\alpha}^{2}}\left(\mathbf{Y}_{i k}-\mu_{\alpha} e^{-\beta / S_{k}} \boldsymbol{\Lambda}\right)\right] \text {, } \\
& E\left(-\frac{\partial^{2} \ell}{\partial \sigma_{\alpha} \partial \beta}\right)=E\left(-\frac{\partial^{2} \ell}{\partial \beta \partial \sigma_{\alpha}}\right)=\sum_{k=1}^{d} \frac{n_{k}}{2} \frac{\partial^{2}\left(\ln \left|\Sigma_{k}\right|\right)}{\partial \sigma_{\alpha} \partial \beta}+E\left[\frac{1}{2}\right. \\
& \left.\cdot \sum_{k=1}^{d} \sum_{i=1}^{n_{k}}\left(\mathbf{Y}_{i k}-\mu_{\alpha} e^{-\beta / S_{k}} \boldsymbol{\Lambda}\right)^{\prime} \frac{\partial^{2}\left(\boldsymbol{\Sigma}_{k}^{-1}\right)}{\partial \sigma_{\alpha} \partial \beta}\left(\mathbf{Y}_{i k}-\mu_{\alpha} e^{-\beta / S_{k}} \boldsymbol{\Lambda}\right)\right] \text {, } \\
& E\left(-\frac{\partial^{2} \ell}{\partial \sigma_{\alpha} \partial \sigma_{B}}\right)=E\left(-\frac{\partial^{2} \ell}{\partial \sigma_{B} \partial \sigma_{\alpha}}\right)=0, \\
& E\left(-\frac{\partial^{2} \ell}{\partial \sigma_{\alpha} \partial \sigma_{\varepsilon}}\right)=E\left(-\frac{\partial^{2} \ell}{\partial \sigma_{\varepsilon} \partial \sigma_{\alpha}}\right)=0, \\
& E\left(-\frac{\partial^{2} \ell}{\partial \beta^{2}}\right)=\sum_{k=1}^{d} \frac{n_{k}}{2} \frac{\partial^{2}\left(\ln \left|\Sigma_{k}\right|\right)}{\partial \beta^{2}}+E\left[\frac{1}{2}\right. \\
& \left.\cdot \sum_{k=1}^{d} \sum_{i=1}^{n_{k}}\left(\mathbf{Y}_{i k}-\mu_{\alpha} e^{-\beta / S_{k}} \boldsymbol{\Lambda}\right)^{\prime} \frac{\partial^{2}\left(\boldsymbol{\Sigma}_{k}^{-1}\right)}{\partial \beta^{2}}\left(\mathbf{Y}_{i k}-\mu_{\alpha} e^{-\beta / S_{k}} \boldsymbol{\Lambda}\right)\right], \\
& E\left(-\frac{\partial^{2} \ell}{\partial \beta \partial \sigma_{B}}\right)=E\left(-\frac{\partial^{2} \ell}{\partial \sigma_{B} \partial \beta}\right)=0 \text {, }
\end{aligned}
$$

$$
\begin{aligned}
& E\left(-\frac{\partial^{2} \ell}{\partial \beta \partial \sigma_{\varepsilon}}\right)=E\left(-\frac{\partial^{2} \ell}{\partial \sigma_{\varepsilon} \partial \beta}\right)=0, \\
& E\left(-\frac{\partial^{2} \ell}{\partial \sigma_{B}^{2}}\right)=\sum_{k=1}^{d} \frac{n_{k}}{2} \frac{\partial^{2}\left(\ln \left|\Sigma_{k}\right|\right)}{\partial \sigma_{B}^{2}}+E\left[\frac{1}{2}\right. \\
& \left.\cdot \sum_{k=1}^{d} \sum_{i=1}^{n_{k}}\left(\mathbf{Y}_{i k}-\mu_{\alpha} e^{-\beta / S_{k}} \boldsymbol{\Lambda}\right)^{\prime} \frac{\partial^{2}\left(\boldsymbol{\Sigma}_{k}^{-1}\right)}{\partial \sigma_{B}^{2}}\left(\mathbf{Y}_{i k}-\mu_{\alpha} e^{-\beta / S_{k}} \boldsymbol{\Lambda}\right)\right], \\
& \left.E\left(-\frac{\partial^{2} \ell}{\partial \sigma_{B} \partial \sigma_{\varepsilon}}\right)=E\left(-\frac{\partial^{2} \ell}{\partial \sigma_{\varepsilon} \partial \sigma_{B}}\right)=\sum_{k=1}^{d} \frac{n_{k}}{2} \frac{\partial^{2}\left(\ln \left|\Sigma_{k}\right|\right)}{\partial \sigma_{B} \partial \sigma_{\varepsilon}}+E\right] \frac{1}{2} \\
& \left.\cdot \sum_{k=1}^{d} \sum_{i=1}^{n_{k}}\left(\mathbf{Y}_{i k}-\mu_{\alpha} e^{-\beta / S_{k}} \boldsymbol{\Lambda}\right)^{\prime} \frac{\partial^{2}\left(\boldsymbol{\Sigma}_{k}{ }^{-1}\right)}{\partial \sigma_{B} \partial \sigma_{\varepsilon}}\left(\mathbf{Y}_{i k}-\mu_{\alpha} e^{-\beta / S_{k}} \boldsymbol{\Lambda}\right)\right], \\
& E\left(-\frac{\partial^{2} \ell}{\partial \sigma_{\varepsilon}^{2}}\right)=\sum_{k=1}^{d} \frac{n_{k}}{2} \frac{\partial^{2}\left(\ln \left|\Sigma_{k}\right|\right)}{\partial \sigma_{\varepsilon}^{2}}+E\left[\frac{1}{2}\right. \\
& \left.\quad \cdot \sum_{k=1}^{d} \sum_{i=1}^{n_{k}}\left(\mathbf{Y}_{i k}-\mu_{\alpha} e^{-\beta / S_{k}} \boldsymbol{\Lambda}\right)^{\prime} \frac{\partial^{2}\left(\boldsymbol{\Sigma}_{k}{ }^{-1}\right)}{\partial \sigma_{\varepsilon}^{2}}\left(\mathbf{Y}_{i k}-\mu_{\alpha} e^{-\beta / S_{k}} \boldsymbol{\Lambda}\right)\right] .
\end{aligned}
$$

The expression of $\mathbf{H}^{\prime}$ is $\mathbf{H}^{\prime}=\left(-\omega / \mu_{\alpha}^{2} e^{-\beta / S_{0}}, 0, \omega / \mu_{\alpha} S_{0} e^{-\beta / S_{0}}\right.$, $0,0)$.

By the delta method, the asymptotic variance of $\widehat{\mathrm{MTTF}}_{0}$ can be calculated by using the following formulation:

$$
\begin{aligned}
& \operatorname{Avar}\left(\widehat{T}_{\mathrm{MTTF}} \mid \xi\right) \\
& =\left(-\frac{\omega}{\mu_{\alpha}^{2} e^{-\beta / S_{0}}}, 0, \frac{\omega}{\mu_{\alpha} S_{0} e^{-\beta / S_{0}}}, 0,0\right) \mathbf{I}^{-1}(\boldsymbol{\theta}) \\
& \quad \cdot\left(-\frac{\omega}{\mu_{\alpha}^{2} e^{-\beta / S_{0}}}, 0, \frac{\omega}{\mu_{\alpha} S_{0} e^{-\beta / S_{0}}}, 0,0\right)^{\prime} .
\end{aligned}
$$

\section{Competing Interests}

The authors declare that there is no conflict of interests regarding the publication of this paper. 
TABLE 5: (a) Optimal three-level CSADT plans under various prefixed budget constraints. (b) Optimal-three level CSADT plans under various duration constraints. (c) Optimal three-level CSADT plans under various sample size constraints.

(a)

\begin{tabular}{lccccccc}
\hline$C_{b}$ & $n_{1}^{*}$ & $n_{2}^{*}$ & $n_{3}^{*}$ & $f^{*}$ & $m^{*}$ & Avar $/ 10^{3}$ & Budgets \\
\hline 1000 & 7 & 5 & 4 & 282 & 3 & 1.926 & 999 \\
1500 & 13 & 10 & 7 & 280 & 3 & 1.031 & 1500 \\
2000 & 16 & 12 & 10 & 278 & 4 & 0.6934 & 2000 \\
2500 & 21 & 16 & 13 & 300 & 4 & 0.5150 & 2500 \\
3000 & 21 & 16 & 13 & 300 & 4 & 0.4303 & 3000 \\
3500 & 21 & 16 & 13 & 200 & 10 & 0.3908 & 3500 \\
4000 & 21 & 16 & 13 & 133 & 15 & 0.3719 & 3997.5 \\
\hline
\end{tabular}

Remark 5: the test duration constraint $t_{A}=2000$ and the sample size constraint $N_{A}=50$.

(b)

\begin{tabular}{lccccccc}
\hline$t_{A}$ & $n_{1}^{*}$ & $n_{2}^{*}$ & $n_{3}^{*}$ & $f^{*}$ & $m^{*}$ & Avar $/ 10^{3}$ & Budgets \\
\hline 500 & 22 & 16 & 12 & 166 & 3 & 0.7538 & 2049 \\
1000 & 21 & 16 & 13 & 200 & 5 & 0.5190 & 2500 \\
1500 & 21 & 16 & 13 & 300 & 4 & 0.5150 & 2500 \\
2000 & 21 & 16 & 13 & 300 & 4 & 0.5150 & 2500 \\
2500 & 21 & 16 & 13 & 300 & 4 & 0.5150 & 2500 \\
3000 & 21 & 16 & 13 & 300 & 4 & 0.5150 & 2500 \\
\hline
\end{tabular}

Remark 6: the prefixed budget constraint $C_{b}=2500$ and the sample size constraint $N_{A}=50$.

(c)

\begin{tabular}{cccccccc}
\hline$N_{A}$ & $n_{1}^{*}$ & $n_{2}^{*}$ & $n_{3}^{*}$ & $f^{*}$ & $m^{*}$ & Avar $/ 10^{3}$ & Budgets \\
\hline 20 & 8 & 7 & 5 & 90 & 22 & 0.8951 & 2470 \\
30 & 12 & 10 & 8 & 200 & 10 & 0.6518 & 2500 \\
40 & 17 & 13 & 10 & 211 & 7 & 0.5497 & 2498.5 \\
50 & 21 & 16 & 13 & 300 & 4 & 0.5150 & 2500 \\
60 & 21 & 16 & 13 & 300 & 4 & 0.5150 & 2500 \\
70 & 22 & 16 & 13 & 281 & 4 & 0.5148 & 2500 \\
\hline
\end{tabular}

Remark 7: the prefixed budget constraint $C_{b}=2500$ and the test duration constraint $t_{A}=2000$.

\section{Acknowledgments}

The authors would like to acknowledge great support by National Natural Science Foundation of China (51475289).

\section{References}

[1] Q. Feng, H. Peng, and D. W. Coit, "A degradation-based model for joint optimization of burn-in, quality inspection, and maintenance: a light display device application," The International Journal of Advanced Manufacturing Technology, vol. 50, no. 5-8, pp. 801-808, 2010.

[2] N. Z. Gebraeel, M. A. Lawley, R. Li, and J. K. Ryan, "Residual-life distributions from component degradation signals: a Bayesian approach," IIE Transactions, vol. 37, no. 6, pp. 543-557, 2005.

[3] H. Liao and E. A. Elsayed, "Reliability inference for field conditions from accelerated degradation testing," Naval Research Logistics, vol. 53, no. 6, pp. 576-587, 2006.
[4] H.-F. Yu, "Designing a screening experiment with a reciprocal Weibull degradation rate," Computers and Industrial Engineering, vol. 52, no. 2, pp. 175-191, 2007.

[5] S.-T. Tseng, N. Balakrishnan, and C.-C. Tsai, "Optimal stepstress accelerated degradation test plan for gamma degradation processes," IEEE Transactions on Reliability, vol. 58, no. 4, pp. 611-618, 2009.

[6] C.-H. Hu, M.-Y. Lee, and J. Tang, "Optimum step-stress accelerated degradation test for Wiener degradation process under constraints," European Journal of Operational Research, vol. 241, no. 2, pp. 412-421, 2015.

[7] C.-Y. Peng and S.-T. Tseng, "Progressive-stress accelerated degradation test for highly-reliable products," IEEE Transactions on Reliability, vol. 59, no. 1, pp. 30-37, 2010.

[8] Z. Pan, N. Balakrishnan, and Q. Sun, "Bivariate constant-stress accelerated degradation model and inference," Communications in Statistics. Simulation and Computation, vol. 40, no. 2, pp. 247257, 2011.

[9] M. S. Hamada, A. Wilson, and C. S. Reese, Bayesian Reliability, Springer, Berlin, Germany, 2008.

[10] S. T. Tseng, C. C. Tsai, and N. Balakrishnan, "Optimal sample size allocation for accelerated degradation test based on Wiener process," in Encyclopedia of Statistical Sciences, John Wiley \& Sons, 2011.

[11] Z. Ye, N. Chen, and K.-L. Tsui, "A Bayesian approach to condition monitoring with imperfect inspections," Quality and Reliability Engineering International, vol. 31, no. 3, pp. 513-522, 2015.

[12] X. Wang, N. Balakrishnan, and B. Guo, "Residual life estimation based on a generalized Wiener degradation process," Reliability Engineering and System Safety, vol. 124, pp. 13-23, 2014.

[13] S. Tang, X. Guo, C. Yu, H. Xue, and Z. Zhou, "Accelerated degradation tests modeling based on the nonlinear wiener process with random effects," Mathematical Problems in Engineering, vol. 2014, Article ID 560726, 11 pages, 2014.

[14] Z.-S. Ye, Y. Wang, K.-L. Tsui, and M. Pecht, "Degradation data analysis using wiener processes with measurement errors," IEEE Transactions on Reliability, vol. 62, no. 4, pp. 772-780, 2013.

[15] S. Tang, C. Yu, X. Wang, X. Guo, and X. Si, "Remaining useful life prediction of lithium-ion batteries based on the wiener process with measurement error," Energies, vol. 7, no. 2, pp. 520547,2014

[16] X. Wang, P. Jiang, B. Guo, and Z. Cheng, "Real-time reliability evaluation with a general Wiener process-based degradation model," Quality and Reliability Engineering International, vol. 30, no. 2, pp. 205-220, 2014.

[17] X. Wang, B. Guo, and Z. Cheng, "Residual life estimation based on bivariate Wiener degradation process with timescale transformations," Journal of Statistical Computation and Simulation, vol. 84, no. 3, pp. 545-563, 2014.

[18] G. A. Whitmore, "Estimating degradation by a wiener diffusion process subject to measurement error," Lifetime Data Analysis, vol. 1, no. 3, pp. 307-319, 1995.

[19] S.-J. Tang, X.-S. Guo, C.-Q. Yu, Z.-J. Zhou, Z.-F. Zhou, and B.C. Zhang, "Real time remaining useful life prediction based on nonlinear Wiener based degradation processes with measurement errors," Journal of Central South University, vol. 21, no. 12, pp. 4509-4517, 2014.

[20] C.-C. Tsai, C.-T. Lin, and N. Balakrishnan, "Optimal design for accelerated-stress acceptance test based on wiener process," IEEE Transactions on Reliability, vol. 64, no. 2, pp. 603-612, 2015. 
[21] N. Balakrishnan and M. H. Ling, "Best constant-stress accelerated life-test plans with multiple stress factors for one-shot device testing under a weibull distribution," IEEE Transactions on Reliability, vol. 63, no. 4, pp. 944-952, 2014.

[22] Z.-S. Ye, L.-P. Chen, L. C. Tang, and M. Xie, "Accelerated degradation test planning using the inverse gaussian process," IEEE Transactions on Reliability, vol. 63, no. 3, pp. 750-763, 2014.

[23] C. Qian, X. J. Fan, J. J. Fan, C. A. Yuan, and G. Q. Zhang, "An accelerated test method of luminous flux depreciation for LED luminaires and lamps," Reliability Engineering \& System Safety, vol. 147, pp. 84-92, 2016.

[24] C. Zhang, X. Lu, Y. Tan, and Y. Wang, "Reliability demonstration methodology for products with Gamma Process by optimal accelerated degradation testing," Reliability Engineering and System Safety, vol. 142, pp. 369-377, 2015.

[25] D. Han, "Time and cost constrained optimal designs of constant-stress and step-stress accelerated life tests," Reliability Engineering and System Safety, vol. 140, pp. 1-14, 2015.

[26] R. Pan, T. Yang, and K. Seo, "Planning constant-stress accelerated life tests for acceleration model selection," IEEE Transactions on Reliability, vol. 64, no. 4, pp. 1356-1366, 2015.

[27] T.-R. Tsai, W.-Y. Sung, Y. L. Lio, S. I. Chang, and J.-C. Lu, “Optimal two-variable accelerated degradation test plan for gamma degradation processes," IEEE Transactions on Reliability, vol. 65, no. 1, pp. 459-468, 2016.

[28] W. Wang and D. B. Kececioglu, "Fitting the Weibull loglinear model to accelerated life-test data," IEEE Transactions on Reliability, vol. 49, no. 2, pp. 217-223, 2000. 


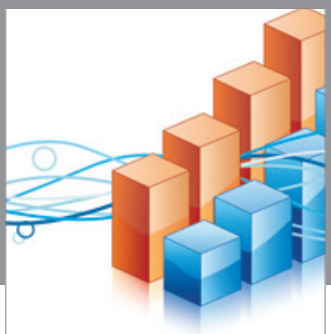

Advances in

Operations Research

vatem alat4

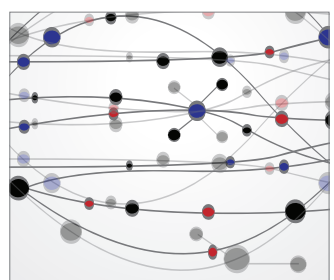

\section{The Scientific} World Journal
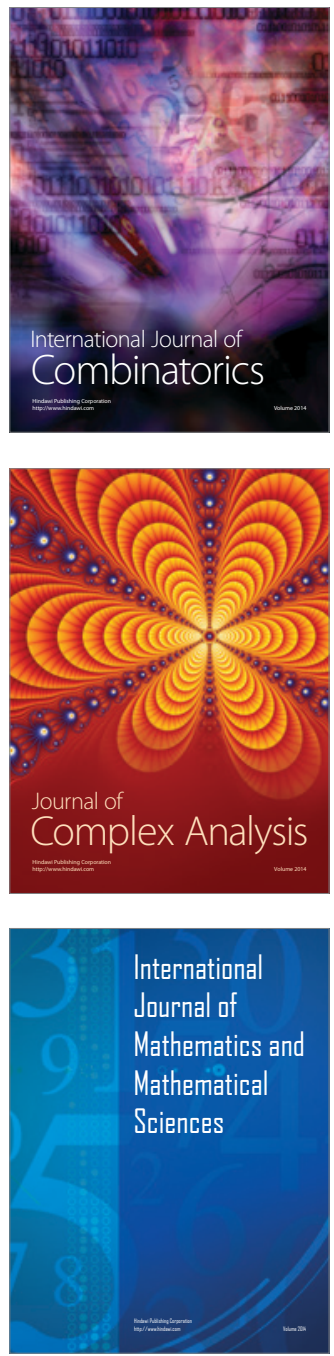
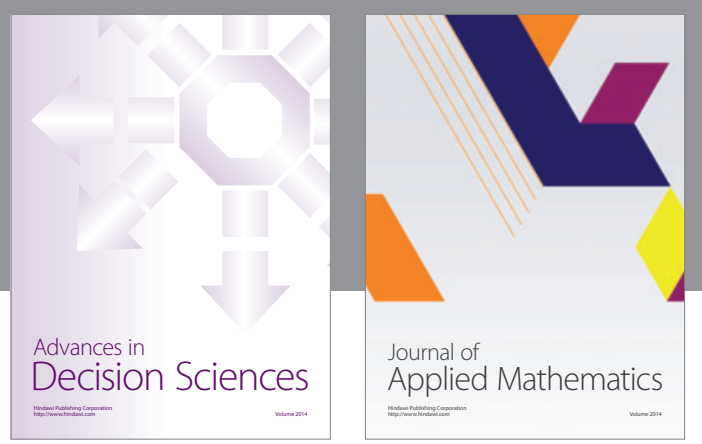

Algebra

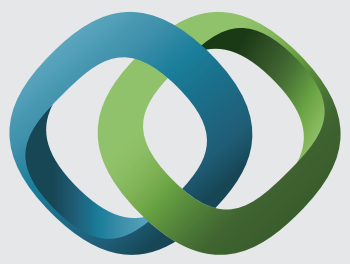

\section{Hindawi}

Submit your manuscripts at

http://www.hindawi.com
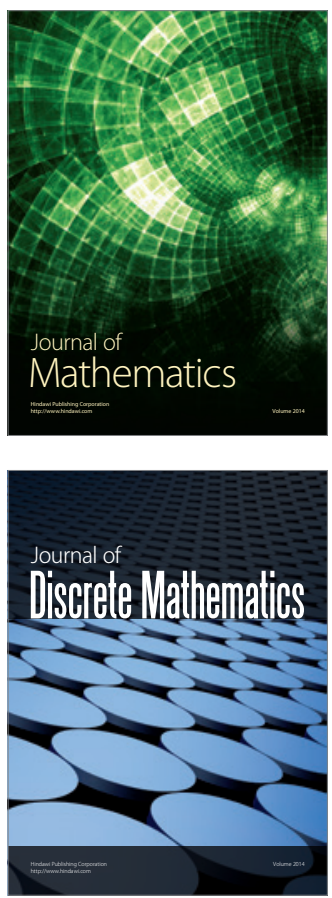

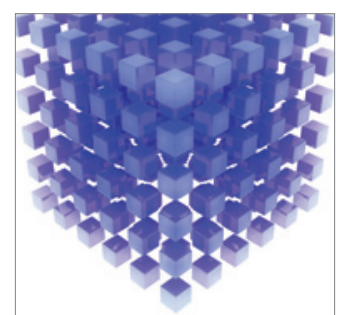

Mathematical Problems in Engineering
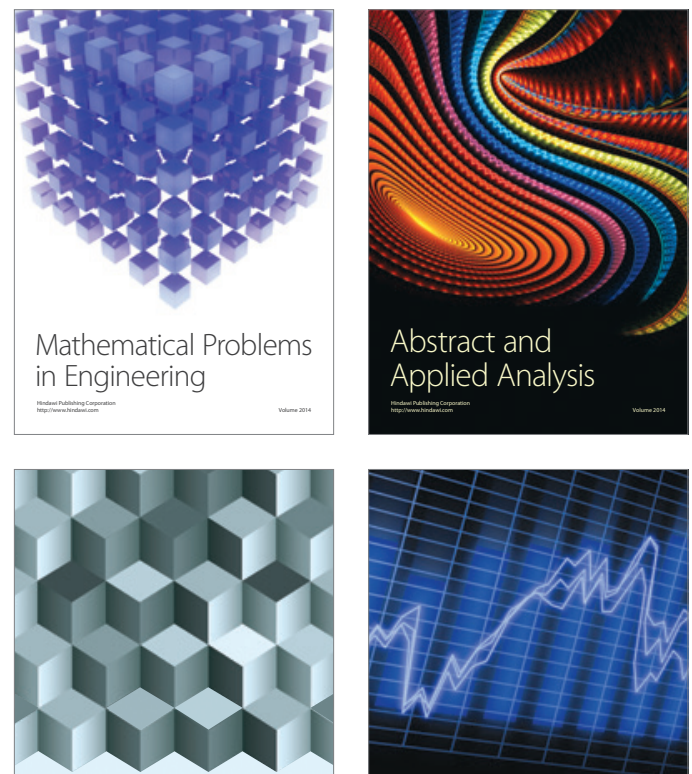

Journal of

Function Spaces

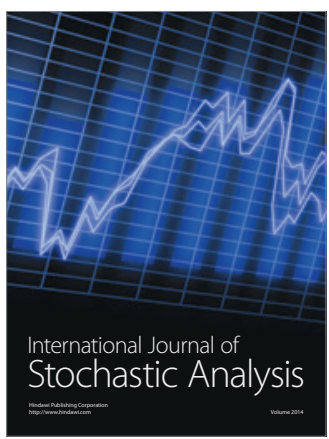

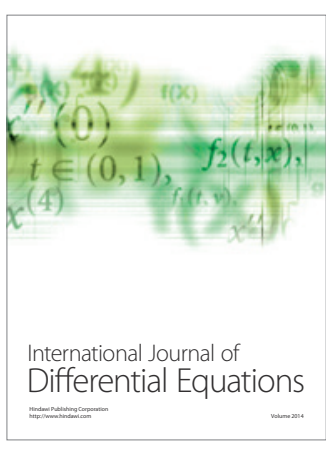
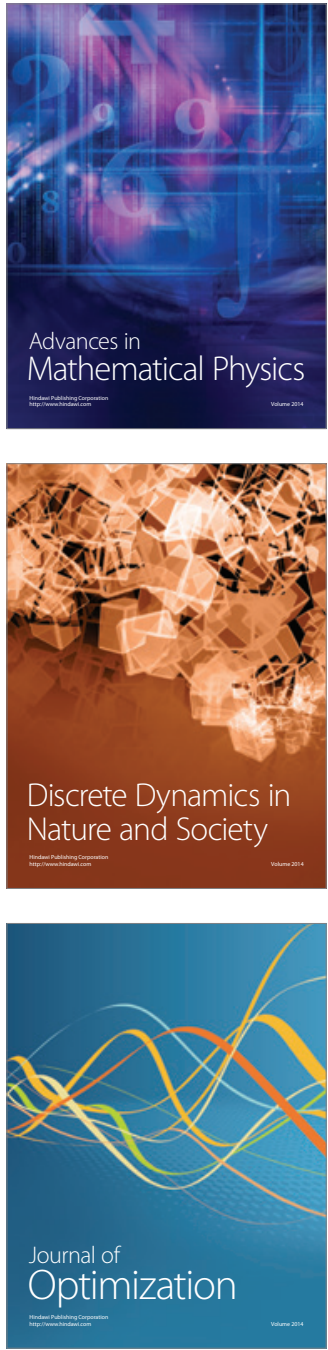